\title{
Art in the Cascade of Brain Areas
}

\section{Petr Adámek}

National Institute of Mental Health (NIMH)

Third Faculty of Medicine Charles University

Dominika Grygarová

National Institute of Mental Health (NIMH)

\begin{abstract}
Over the last three decades, we have witnessed the gradual penetration of modern and often experimental technologies into the humanities, which often could only prove their conclusions within the framework of logic and logical derivation. This trend can also be observed in fields such as aesthetics and art history. Experimental devices currently help us understand and describe events in our brain that contribute, for example, to why some works of art appeal to us and others do not. They help us to describe the techniques used by the artist to change our perception of his work and thus evoke certain emotions in us. This article should provide the reader with a basic orientation in the relatively young field of neuroesthetics and at the same time introduce the current state of the field in the environment of the Czech Republic.
\end{abstract}

\section{Keywords}

Neuroscience; art; aesthetics; experiment; humanities.

\section{Contact address}

Národní ustav duševního zdraví (National Institute of Mental Health), Topolová 748, 25067 Klecany, Czechia. 
Neuroaesthetics, a cognitive neuroscience of art, is seen as one of many subdisciplines in the neuroscience framework. Up to recently, we have been navigating within imprecisely defined limits of broader neuroscience research. Apart from the effort to explain actions taking place in viewers when perceiving a work of art, we need to define what various approaches to neuroaesthetics have in common. Each of them has evolved and adopted different methods and defined the different essential questions they investigate.

Efforts to study what goes on in viewers when they perceive an object as aesthetically appealing can be observed from the times of British Empiricism. Emotions, music, but also aesthetic feeling were explained as creating a vibration of human nerves shaking "moving spirits", ${ }^{1}$ which then mediated mental conditions both positive and negative. ${ }^{2}$ According to the British Empiricists, experienced emotions could also be indicated by a change in nerve fibres and their reverse transgression to a normal resting state. ${ }^{3}$ These approaches lacked both experimental and quantitative evaluation methods of verification. The birth of empiric aesthetics and its methodology represents a crucial turn in the development of neuroaesthetics. The first to attempt this approach was Gustav Fechner, who introduced a method used to this day measuring preferences in a large group of responders. He tried to suppress the individualized view by an individual of a piece of art and create a broader, in principle objectified, view of "the general majority" in a particular cultural and diachronic frame of human community. He compared the collected data with individual characteristic features of artworks aiming at creating general categories influencing the aesthetic perception of the object at stake. ${ }^{4}$ Gestalt psychology stood in a certain opposition to this reductionist approach to works of art and criticized it. ${ }^{5}$ Contrary to this earlier approach, it asked how the overall arrangement of individual elements in the structure of a scene influenced the final perception in the viewer and how it is then processed in dynamic interaction (fig. 1) ${ }^{6}$. The viewer ceased to be a passive recipient, and became an active agent influencing his/her experience.

One of the recent significant updates in methodological approaches to the issue was engaging the elements of viewer's motivation and emotions in the equation of the overall

\footnotetext{
1 "Esprits animaux" (animal spirits) is a mechanistic construct of excitation-transfer to perception, which was prevalent in modern philosophy (René Descartes, Nicolas Malebranche, David Hume, Baruch Spinoza, etc.). Its tradition reaches back, however, to ancient medicine (Galen etc.).

${ }^{2}$ Daniel Webb, Observations on the Correspondence between Poetry and Music (J. Dodsley, 1769).

${ }^{3}$ Uvedale Price, Essays on the Picturesque, as Compared with the Sublime and the Beautiful, and, on the Use of Studying Pictures, for the Purpose of Improving Real Landscape, vol. 2 (J. Mawman, 22, Poultry., 1810).

${ }^{4} \mathrm{He}$ focused mainly on rhythm, contrast, shape, colour, symmetry, etc. See, Gustav Theodor Fechner, Vorschule der aesthetik, vol. 1 (Breitkopf \& Härtel, 1876).

${ }^{5}$ Gestalt psychology was originally interested in the general problem of visual perception and how perception is subsequently processed in our mind. Rudolf Arnheim, in particular, is to be credited for the transfer of methodological principles to pieces of art. We also find gestalt principles as a theoretical base for many of today's works on neuroaesthetics.

${ }^{6}$ All images used on these article belong to Public domain copyright or CC BY-SA 3.0 copyright.
} 

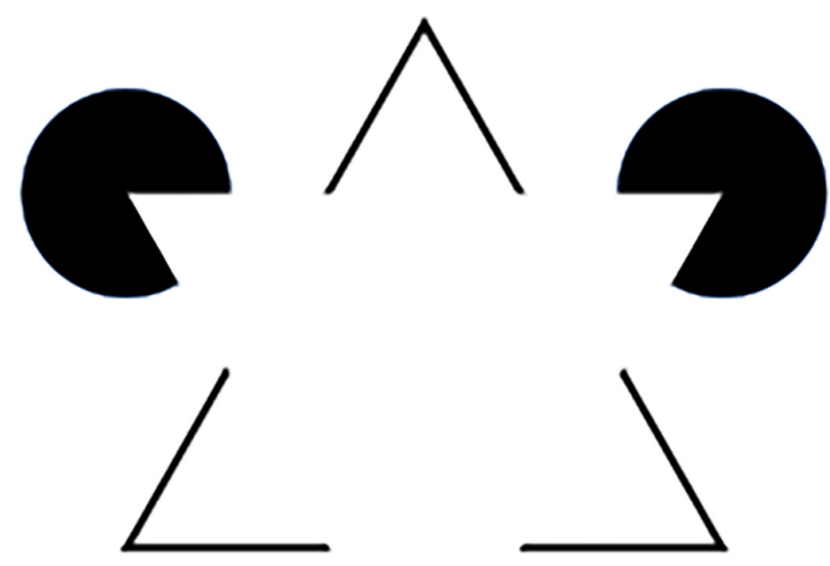

Figure 1. Kanizsa’s triangle: visual perception is based on objective experience. The shape of white triangle is not present in the image, but still is distinguishable.

aesthetic experience. ${ }^{7}$ We have now moved significantly from Fechner's research of formal aspects of art works only, inducing aesthetic pleasure, to the question of the way the viewer organizes and perceives a work of art. It is, for example, a feeling of novelty or ambiguity, ${ }^{8}$ which is not an objective aspect of a piece of art, but a characteristic response of a specific viewer to a specific object. An extraordinary shift in this issue, which was an equally significant novelty in Fechner's day, involving the use of statistical methods in examining pieces of art, was the engagement of experimental devices, which are capable of measuring biological responses to specific works of art. The initial enthusiasm coming from new experimental possibilities gave rise to the first wave of neuroaesthetic studies, which, unfortunately, led to later skepsis on the part of many researchers towards this new field. The emphasis on primarily evolutionary psychological (and thus a still theoretical, not experimental) approach, which located the aesthetic experience among existing knowledge of cognitive psychology and evolutionary biology findings, was seen as the main pitfall. The most renowned representative of this movement is Vilayanur Ramachandran. ${ }^{9} \mathrm{He}$, as well as a number of other authors, interpreted aesthetic feeling as one of the tools of natural selection. From the perspective of a historian of art, one of their amusing reductive

7 Paul J Silvia, "Emotional Responses to Art: From Collation and Arousal to Cognition and Emotion," Review of General Psychology 9, no. 4 (2005).

8 The dimension of "novelty" and "ambiguity" falls under the so-called "collative" properties of a work of art, which are spontaneously connected and enter the viewer's perception configuration together in Daniel E Berlyne, "Aesthetics and Psychobiology," (1973). Berlyne placed, for example, excitement/ arousal, novelty and the hedonic tone among those.

${ }^{9}$ Vilayanur S Ramachandran and William Hirstein, "The Science of Art: A Neurological Theory of Aesthetic Experience,” Journal of Consciousness Studies 6, no. 6-7 (1999). 
interpretations is the theory, in which a work of art is considered an external phenotype of the author or the owner of the art piece. ${ }^{10}$ The author or owner of the piece of art demonstrates its superiority to other individuals in their circle and obtains a certain advantage in the struggle for a partner. As demonstrated, this first wave tried to theoretically explain why and under which circumstances aesthetic response to a certain type of visual objects' arrangement developed in the human mind. This is a topic which is not particularly relevant for historians or art theoreticians. It is even felt to be a somewhat offensive statement, since it links the achievements of human culture, skills and creativity to the domains of primitive instincts. The main problem, however, is their primarily theoretical-speculative approach, grounded in neural determinism, while the "laws of art" are not usually verified in experiment and are based a great deal on generalization and reductionism. This approach is led by a confident conviction that there is just one and final definition of the phenomenon of art as modelled by the natural sciences. Semir Zeki's research ${ }^{11}$ already had experimental grounds ${ }^{12}$ which initially focused on neurobiological aspects of vision and their relationship to the phenomenon of the artistic medium and subsequent artistic experience. Both Ramachandran and Zeki attempted to determine universal rules in the early times of neuroaesthetics, which should have been responsible for a certain object becoming art, and at the same time successful art. ${ }^{13}$

The following development in the area of neuroscience research involves exponential growth of studies and shifts to more rigorous procedures and patient questioning. Research does not claim to bring any grand explanations of art creation as such, but aims at gradually answering more modest and realistic questions through individual experiments. These approaches still try to describe principles ruling the perception of works of art and mechanisms interfering with this complex process. Our multidisciplinary lab, led by Ladislav Kesner, is also part of this stream of various methods and interests. Apart from art historians, psychologists, neuroscientists, mathematicians and data analysts work for the team. We analyse neural and psychological mechanisms taking part in the origin of experience while watching visual works of art, but also different types of images, for example media, both theoretically and experimentally. ${ }^{14} \mathrm{We}$ focus especially on the areas of semantic processing, empathic and affective response which we study by means of functional magnetic resonance

${ }^{10}$ Geoffrey F Miller, "Aesthetic Fitness: How Sexual Selection Shaped Artistic Virtuosity as a Fitness Indicator and Aesthetic Preferences as Mate Choice Criteria," Bulletin of Psychology and the Arts 2, no. 1 (2001).

${ }^{11}$ Semir Zeki, "Inner Vision: An Exploration of Art and the Brain," (2002).

${ }^{12}$ Using predominantly the methods of magnetic resonance.

${ }^{13}$ As concerns the criticism of reductive approaches of the first wave of neuroaesthetics, for example Ladislav Kesner, Neuroaesthetics: Real Promise or Real Delusion?, ed. Ondřej Dadejdík-Jakub Stejskal, The Aesthetic Dimension of Visual Culture, (Cambridge: Cambridge Scholars Publishing, 2010).

${ }^{14}$ Petr Adámek et al., "Tracking Artistic Transformations: Comparing Paintings and their Source Photographs," Ceskoslovenska Psychologie 63, no. 3 (2019); Dominika Grygarová et al., "Illuminating Smiles and Frowns: Visual-Affective Cueing Influences Viewer Perceptions of Page Layout Images," Perceptual and Motor Skills 127, no. 1 (2020); Ladislav Kesner, "Mental Ill-health and the Epidemiology of Representations," Frontiers in Psychiatry 9 (2018); Kesner, Neuroaesthetics: Real Promise or Real Delusion; Ladislav Kesner et al., "Fusiform Activity Distinguishes between Subjects with Low and High Xenophobic Attitudes toward Refugees," 

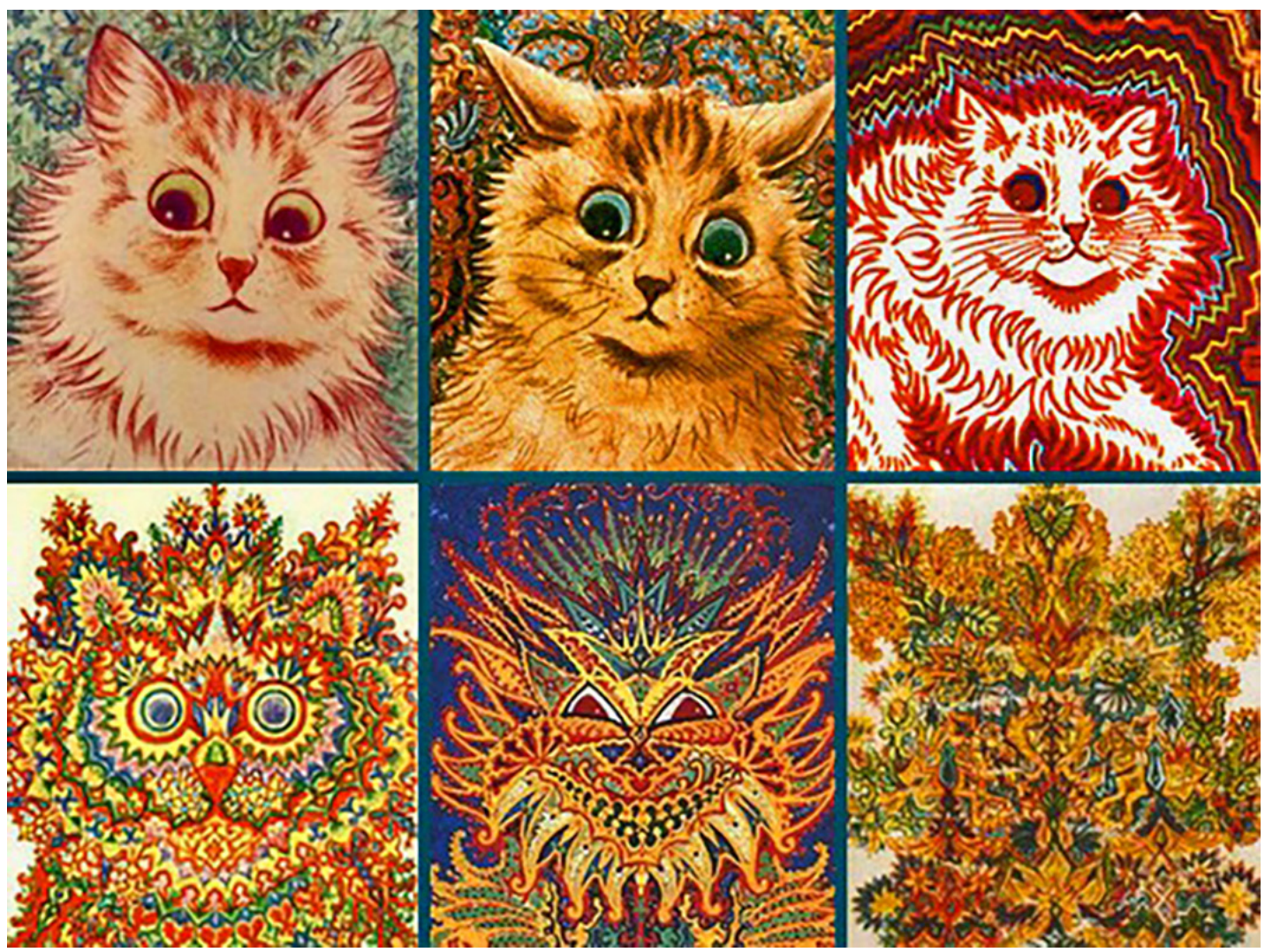

Figure 2. Louis-Wain - Pictures of cats and their changes with disease progression.

imaging, eye tracking and psychological questioning. Our subjects come from the general population, but also from specialists and those diagnosed with psychiatric problems, who help us understand abnormalities or differences in these mechanisms (e.g. viewers with psychiatric diseases, certain type of expertise or cultural knowledge, etc.).

Visual perception reaches our visual cortex through the eye and peripheral pathways of the optic tract, where it is then processed in an entire cascade of brain areas. They evaluate it and, depending on its nature, determine what will happen next - for example, where our attention will go next. The whole mechanics of it are not as simple as it might appear at first sight: there are many processes taking place simultaneously in each phase, biological (e.g. physical salience), ${ }^{15}$ chemical (e.g. misbalance of certain substances in the system, drug use, psychosis), or psychological (mental state, cultural learned patterns, personal

Frontiers in Behavioral Neuroscience 14 (2020); Ladislav Kesner et al., "Perception of Direct vs. Averted Gaze in Portrait Paintings: An fMRI and Eye-tracking Study," Brain and Cognition 125 (2018).

${ }^{15}$ It deals with physical aspects of visual perception attracting attention (contrast, colour, movement, etc.). The physical salience cannot be usually suppressed and in early stages of visual perception is present in all healthy individuals. 


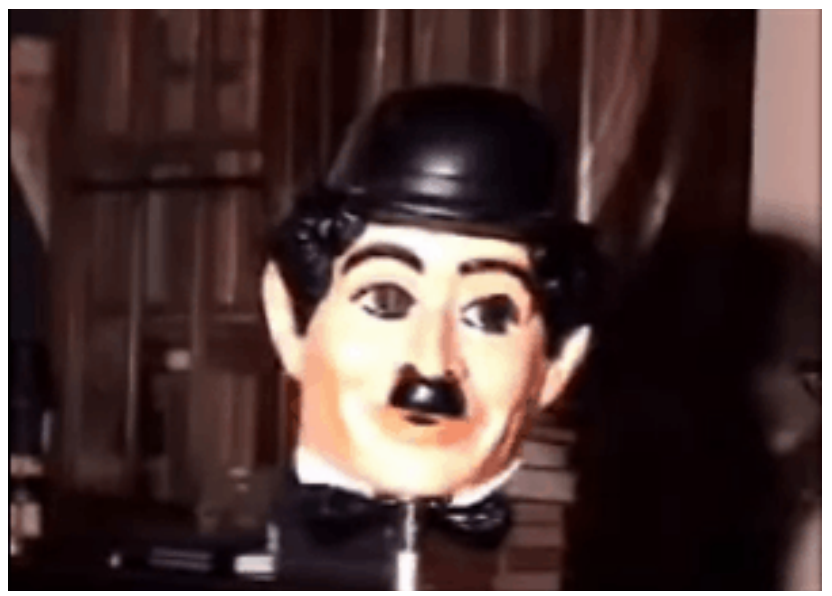

Figure 3. Hollow mask - The face constantly appears as if he was facing us even when he is in the current concave position. Patients with perceptual disorders however, organizations do not succumb to the illusion.

preferences, etc.) which fundamentally influence the final outcome of our perception. In order to understand the functioning of the whole complex phenomenon of vision, the study of patients after a specific injury or with a specific mental disease helps as well. One of the model cases is schizophrenia, which, for example, disrupts and alters organization ${ }^{16}$ of visual perception, and can probably even influence the style and signature of an artist with its progression (fig. 2). One of the main markers of the disease in its early stages, which is often present already prior to the first outbreak, is disruption of smooth eye movements. ${ }^{17}$ Later, there are also external symptoms of diminished ability of contextual evaluation of visual perception and its elements (fig. 3), as well as suppression of the ability to perceive contrasts in a scene. Characteristic suppression of the ability to perceive visual illusions is also bound to the last two visual deficits (fig. 3) ${ }^{18}$ As demonstrated, individual - also basal elements of a scene - can influence overall interpretation of what we see or how we perceive what we see.

These findings have become a pivotal point of one of our earlier research project which focused on artist's manipulation with basic visual properties of a scene and the response of the viewer to this manipulation in a trajectory of his/her eye movements. ${ }^{19}$ By means of recording viewers' eye movement, we determined that viewers in the studied examples

\footnotetext{
${ }^{16}$ Perception organization can be defined as: "processes by means of which individual elements of sensoric information of perceived objects and relationships between them are jointly structured to coherent wholes. Famous gestalt principles like the law of similarity, proximity, continuity or connectedness." (adapted from: Martin Paštrnák, Aneta Dorazilová, and Mabel Rodriguez, "Vizuální percepce a její narušení u schizofrenního onemocněnípřehledová studie," Ceskoslovenska Psychologie 61, no. 6 (2017).

${ }^{17}$ It is an eye movement watching a moving object, this movement is not smooth in schizophrenia, but the eye jumps from one fixation to another.

${ }^{18}$ Charles-Edouard Notredame et al., "What Visual Illusions Teach us about Schizophrenia," Frontiers in Integrative Neuroscience 8 (2014).

${ }^{19}$ Adámek et al., "Tracking Artistic Transformations: Comparing Paintings and their Source Photographs."
} 
actually watched different spots in paintings, than in source photographs (based on which artists created their works). We further compared these differences in viewers' eye movements with an explicit focus by the artist - focusing on spots which the artist marked as essential for realizing his/her intention. The pattern, which was experimentally confirmed in several cases, therefore looks as follows: a painter creates a new painting according to a photograph (we can also substitute a perceived scene or any other visual inspiration an artist decides to process). The painting contains certain visual qualities like colour, contrast, composition, depth of sharpness, the number of objects in a scene and their shapes, etc. All the listed elements or their arrangement (visual differentiation from the background, attracting viewers' attention - "physical salience") can influence where viewer's attention will be directed in the first seconds of viewing the scene. The attention will be captured by the spot with dominant colours (as opposed to a dimmed background), from which further observation will be derived. Naturally, artists do not transfer the original elements from the photograph to canvas in an unchanged form and always want to emphasize or suppress something. Even hyper-realistic painting displays minor deviations from original photography in visual properties. Through this way, by means of created deviations, the painter is capable of guiding viewer's attention and navigating him/her to spots which carry meaning or cause the intended aesthetic effect - thus spots the author wanted to emphasize and prioritize to the viewer's perception. This mechanism can also be related to art theory where this question has resonated for decades, searching for the (im)possibility of artistic intention and the ways of its penetration into a work of art. ${ }^{20}$ Our theoreticalexperimental suggestion reveals the artist's intentions purely from information we learn from the viewer's perspective: perceived specific spots in a painting recorded by means of measured eye movements (the difference between a record of eye movement when viewing a photograph and a resulting painting). We consider it an implicit index of artist's creative intention, which we understand as a set of not only mental and conscious, but also unconscious, interventions to the final structure of a piece of art. ${ }^{21}$ There were several pieces of art that we studied, where we were able to state that viewers' behaviour on the attention level reflected traces of the artist's intentions even in the traditional meaning of the word, when intention is only a thoughtful and conscious layer by means of statements of artists. In the following studies, we try to confirm a theory that if the author of the painting can attract viewers' attention at the beginning of his/her oculomotor way through the painting to spots with a certain meaning-bearing value, its subsequent viewing will be influenced by this entry point, or as a result also his/her affective or empathic response to the painting. ${ }^{22}$

\footnotetext{
${ }^{20}$ David Summers, "Intentions in the History of Art," New Literary History 17, no. 2 (1986). Mark Rollins, What Monet Meant. Intention and Attention in Understanding Art, The Journal of Aesthetics and Art Criticism LXII, 2004, pp 175-188; etc.

${ }^{21}$ See Elisabeth Pacherie, "Towards a Dynamic Theory of Intentions," Does Consciousness Cause Behavior (2006).; Alessandro Pignocchi, "How the Intentions of the Draftsman Shape Perception of a Drawing," Consciousness and Cognition 19, no. 4 (2010).

${ }^{22}$ Grygarová et al., "Illuminating Smiles and Frowns: Visual-Affective Cueing Influences Viewer Perceptions of Page Layout Images."
} 

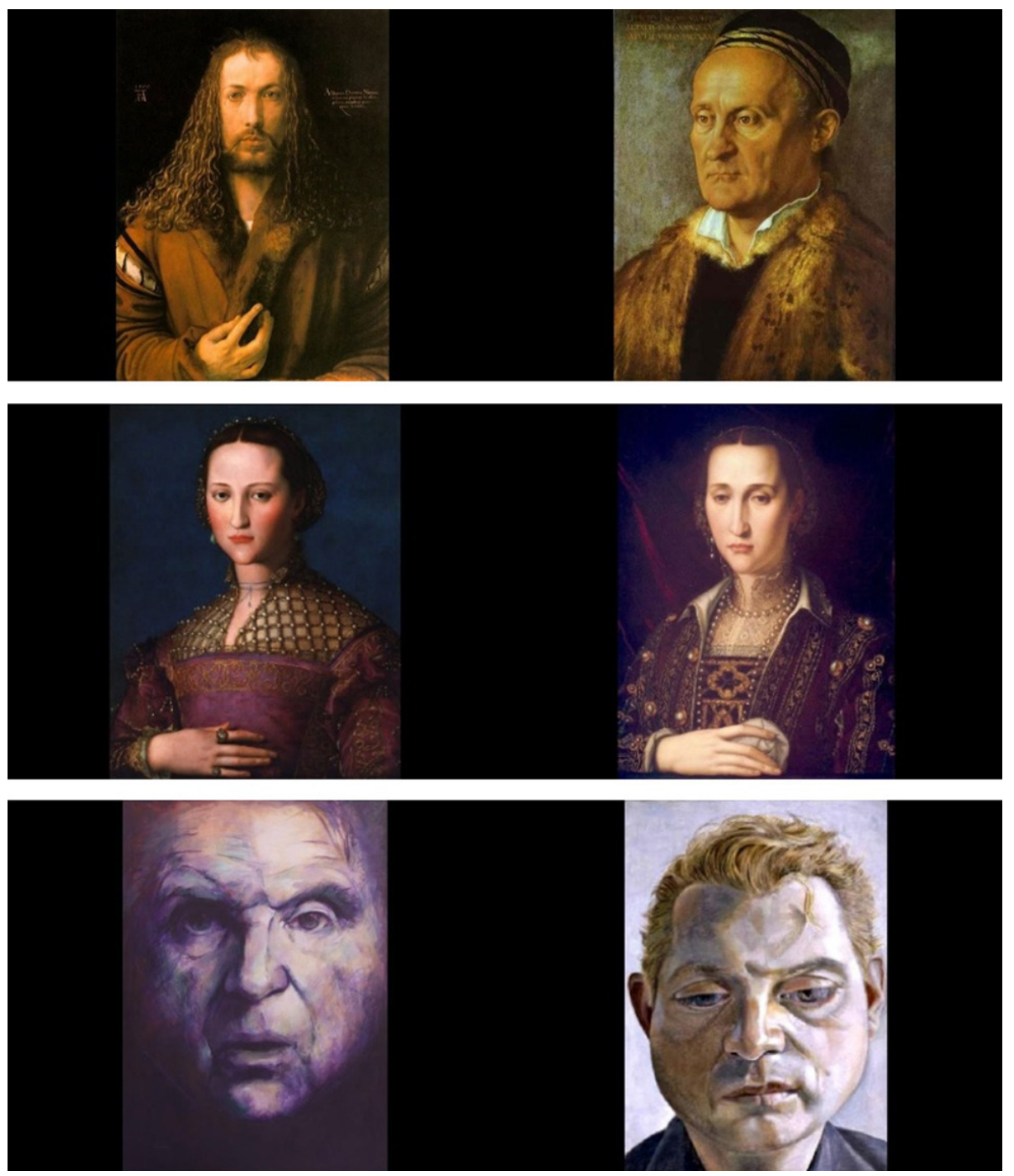

Figure 4 .

Sample of stimulatory set

One of other studies ${ }^{23}$ by our research team focused on the question of gaze (regard) as one of the main art history terms expressing the communication between the art piece and the active reception of a viewer. The regard ( gaze) term was introduced to the discourse of visual culture and history of art by the psychoanalyst Jacques Lacan. ${ }^{24}$ In his view, a gaze does not only belong to the one who is watching, but also to the object the viewer is regarding. A gaze is a shared process of watching between the viewer and the watched, an object thus invites a viewer to a certain gaze. Lacan in his regard theory, counts on certain inherent properties of the object itself that set limits to our perception of it. In other words, a concrete arrangement of the object manipulates us to this set-up relationship, to

${ }^{23}$ Kesner et al., "Perception of Direct vs. Averted Gaze in Portrait Paintings: An fMRI and Eye-tracking Study." ${ }^{24}$ Jacques Lacan, The Split between the Eye and the Gaze (2003). 
a specific way and engagement of gaze. We decided to subject the question of gaze of a portrayed person to controlled experimental conditions: a set of 72 portraits was assembled in pairs of similar portraits by the same author, always one with a direct gaze at a viewer and one with an averted gaze (fig. 4). The set was shown to 24 healthy individuals without education in art in a functional magnetic resonance imaging experiment. The results of the analysis showed several interesting findings. When comparing direct against indirect gaze, the area of fusiform gyrus, which is generally considered the main area for processing of human faces, was more activated. It was also further described as a structure of the brain which enables deeper processing and coding of faces. Greater engagement of this area under the condition of direct gaze as opposed to averted gaze could have originated on the basis of volitional attention, when viewers paid more attention to monitoring the eyes and lips of the portrayed person (elements from which we derive the intention and state of mind of a figure in portrait), which was also confirmed by our eye-tracking data. This "facial area" is located in temporal lobe together with another area (angular gyrus), which is also activated while watching direct as opposed to indirect gazes, associated with social cognition and with inference of the mental states of the depicted person. Two other regions, activated with direct as opposed to averted gaze (in the lower and medium part of the prefrontal lobe), are associated with the so-called theory of mind, which describes the ability to read extremely quickly the state of mind and intentions of the second person only from visual indications, e.g. from facial expression and body posture. A recent study even found a connection between this area with communicative intention and readiness for a response with a living person. ${ }^{25}$ There is no more space for naming further concrete findings of this study, but let us at least enumerate basic findings, which we can relate to the aesthetics and history of art. The experiment confirms that a direct gaze of portrayed persons actually forced viewers to a more socially-engaged gaze, made them more interested in looking into person's eyes and at his/her lips, and implicitly alarmed viewers to potential communication interaction similarly as with a living person; all this in contrast to a response to very similar portrayed faces not looking directly at viewers. An advantage of the experimental approach is primarily rigorous empirical evidence: a conclusion on social activation of a viewer's gaze at persons with a direct gaze can be observed despite the fact that the collected data of many subjects had strict rules applied for calculation of statistical significance which govern all scientific studies - by means of which a drug is evaluated in drug trials to see if it is effective or if there is a non-significant correlation between a drug and the positive response of a user. Another advantage is that it is possible to obtain information about the reaction of a viewer which does not have to be conscious, but still affects viewers' response or opinion.

There is a fundamental connection between the two presented studies apart from the common denominator - pieces of art as research stimuli. Both are derived from theoretical models originating both in neuroscience and art theory. Neuroscientific research does not

${ }^{25}$ Andrea Cavallo et al., "When Gaze Opens the Channel for Communication: Integrative Role of IFG and MPFC," NeuroImage 119 (2015). 
ignore questions and findings of humanities research with their own universal theories as was the case at the beginning, but attempts to widen the perspective of the humanities and tries to support them by findings from natural sciences. This is the only way to achieve meaningful results with general validity.

\section{Bibliography}

Adámek, Petr, Dominika Grygarová, Jiří Lukavský, and Ladislav Kesner. "Tracking Artistic Transformations: Comparing Paintings and Their Source Photographs." Ceskoslovenska Psychologie 63, no. 3 (2019). DOI: 10.1016/j.neuroimage.2015.06.025

Berlyne, Daniel E. "Aesthetics and Psychobiology." (1973).

Cavallo, Andrea, Ovidiu Lungu, Cristina Becchio, Caterina Ansuini, Aldo Rustichini, and Luciano Fadiga. "When Gaze Opens the Channel for Communication: Integrative Role of Ifg and Mpfc." NeuroImage 119 (2015): 63-69.

Fechner, Gustav Theodor. Vorschule Der Aesthetik. Vol. 1: Breitkopf \& Härtel, 1876. DOI: 10.1017/ СВO9781139854580

Grygarová, Dominika, Petr Adámek, Jiř́ Lukavský, and Ladislav Kesner. "Illuminating Smiles and Frowns: Visual-Affective Cueing Influences Viewer Perceptions of Page Layout Images." Perceptual and Motor Skills 127, no. 1 (2020): 75-97. DOI: 10.1177/0031512519876742

Kesner, Ladislav. "Mental Ill-Health and the Epidemiology of Representations." Frontiers in psychiatry 9 (2018): 289. DOI: $10.3389 /$ fpsyt.2018.00289

Kesner, Ladislav. Neuroaesthetics: Real Promise or Real Delusion? The Aesthetic Dimension of Visual Culture. Edited by Ondřej Dadejdík-Jakub Stejskal. Cambridge: Cambridge Scholars Publishing, 2010. DOI: 10.3389/fnbeh.2020.00098

Kesner, Ladislav, Iveta Fajnerová, Petr Adámek, Martin Buchtík, Dominika Grygarová, Jaroslav Hlinka, Pavel Kozelka, et al. "Fusiform Activity Distinguishes between Subjects with Low and High Xenophobic Attitudes toward Refugees." Frontiers in Behavioral Neuroscience 14 (2020): 98.

Kesner, Ladislav, Dominika Grygarová, Iveta Fajnerová, Jiř́ Lukavský, Tereza Nekovárová, Jaroslav Tintěra, Yuliya Zaytseva, and Jiř́ Horáček. "Perception of Direct Vs. Averted Gaze in Portrait Paintings: An Fmri and Eye-Tracking Study." Brain and Cognition 125 (2018): 88-99. DOI: 10.1016/j.bandc.2018.06.004

Lacan, Jacques. The Split between the Eye and the Gaze. 2003.

Miller, Geoffrey F. "Aesthetic Fitness: How Sexual Selection Shaped Artistic Virtuosity as a Fitness Indicator and Aesthetic Preferences as Mate Choice Criteria." Bulletin of Psychology and the Arts 2, no. 1 (2001): 20-25.

Notredame, Charles-Edouard, Delphine Pins, Sophie Deneve, and Renaud Jardri. "What Visual Illusions Teach Us About Schizophrenia." Frontiers in Integrative Neuroscience 8 (2014): 63. DOI: 10.3389/fnint.2014.00063

Pacherie, Elisabeth. "Towards a Dynamic Theory of Intentions." Does Consciousness Cause Behavior (2006): 145-67. DOI: $10.7551 / \mathrm{mitpress} / 9780262162371.003 .0009$

Paštrnák, Martin, Aneta Dorazilová, and Mabel Rodriguez. "Vizuální Percepce a Její Narušení U Schizofrenního Onemocnění-Přehledová Studie.” Ceskoslovenska Psychologie 61, no. 6 (2017): 593-604.

Pignocchi, Alessandro. "How the Intentions of the Draftsman Shape Perception of a Drawing." Consciousness and Cognition 19, no. 4 (2010): 887-98. DOI: 10.1016/j.concog.2010.04.009 
Price, Uvedale. Essays on the Picturesque, as Compared with the Sublime and the Beautiful, and, on the Use of Studying Pictures, for the Purpose of Improving Real Landscape. Vol. 2: J. Mawman, 22, Poultry, 1810.

Ramachandran, Vilayanur S, and William Hirstein. "The Science of Art: A Neurological Theory of Aesthetic Experience." Journal of Consciousness Studies 6, no. 6-7 (1999): 15-51.

Silvia, Paul J. "Emotional Responses to Art: From Collation and Arousal to Cognition and Emotion." Review of General Psychology 9, no. 4 (2005): 342-57. DOI: 10.1037/1089-2680.9.4.342

Summers, David. "Intentions in the History of Art." New Literary History 17, no. 2 (1986): 305-21. DOI: $10.2307 / 468898$

Webb, Daniel. Observations on the Correspondence between Poetry and Music. J. Dodsley, 1769.

Zeki, Semir. "Inner Vision: An Exploration of Art and the Brain." (2002). 